\title{
MEMS Gyroscope Control Systems for Direct Angle Measurements
}

\author{
Chien-Yu Chi \\ Mechanical Engineering \\ National Chiao Tung University \\ Hsin-Chu, Taiwan (R.O.C.) 30010 \\ Email: chienyu.me93g@nctu.edu.tw
}

\author{
Tsung-Lin Chen $\dagger$ \\ Mechanical Engineering \\ National Chiao Tung University \\ Hsin-Chu, Taiwan (R.O.C.) 30010 \\ Email: tsunglin@mail.nctu.edu.tw \\ Telephone: +886-3-571-2121 $\sharp 55127$ \\ Fax: +886-3-572-0634
}

\begin{abstract}
This paper presents a control algorithm for vibrating gyroscopes so that they can directly measure the rotation angle without integrating the angular rate. In most gyroscope systems, the rotation angles were obtained by integrating the angular rate, thus suffer from the error accumulation problem. Only a few papers reported that they could compensate the imperfect dynamics in gyroscopes and obtain the rotation angle directly. However, they either required a calibration phase prior to the normal operation or their stability was not theoretically proven. This paper proposes a one-step control algorithm so that imperfection compensation and angle measurement can be done simultaneously. In a demonstrating case, the mechanical structure uncertainties caused $10 \% \sim 20 \%$ parameter variations in a gyroscope system; the signal are contaminated by zero-mean white noise with the PSD of $2.3 \times 10^{-15}\left(\mathrm{~ms}^{-1}\right)^{2} / \mathrm{Hz}$; the angular rate to be measured is $4 \sin (2 \pi \times 40 t) \mathrm{rad} / \mathrm{sec}$. The proposed algorithm can obtain the rotation angle with an accuracy less than 0.5 degree.
\end{abstract}

\section{INTRODUCTION}

MEMS gyroscopes are typically designed to be angular rate sensors, and ideally, the rotation angle can be obtained by integrating the angular rates. In practice, the bias and noise existed in the measured angular rates cause the calculated angles to drift over time [1]. This error accumulation problem can be apprehended by an example shown in Fig. 1. The plot on the top shows a random signal with zero mean. Integrating this signal over time produces a "random walk" signal shown as one line in the middle plot. If this integration process is carried out several times (in this case, 10 zero-mean random signals which lead to 10 random walk signals), one can show that the standard deviation of the random walk signal increases with time as shown in the bottom plot.

In 1978, Friedland and Hutton [2] proposed equations for the direct angle measurements for vibratory gyroscopes, which could avoid the error accumulation problem. However, those equations were applicable only to gyroscopes with "ideal" dynamics, wherein the proof mass of a gyroscope can consistently oscillate along two axes at the same resonant frequency. In that case, the precession angle of the proof mass trajectory, induced by the Coriolis force, equals to the rotation angle to be measured [2] [3] (see Fig. 2). This precession angle can be calculated by the instantaneous values of the proof mass position and velocity.
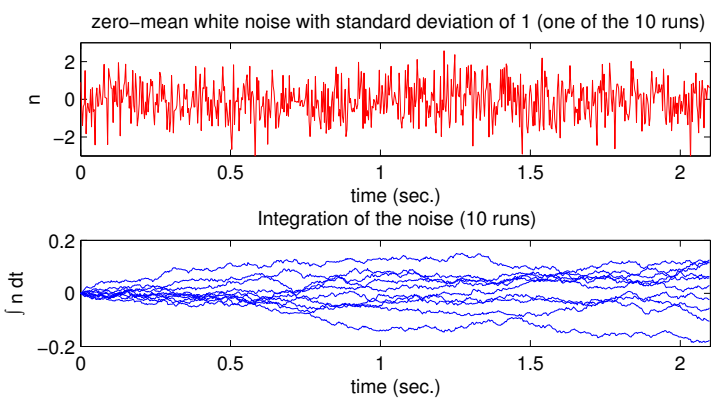

Unbiased STD over runs (10 runs)

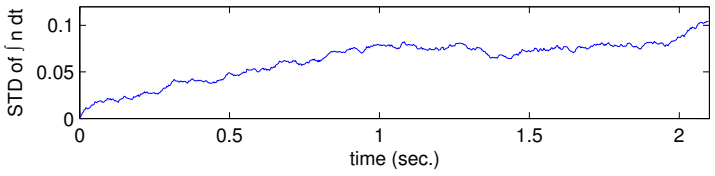

Fig. 1. Signal drifts due to integrating the zero-mean white noise. Ten random signals are generated and integrated over time. The top plot shows one of the ten signals; the middle plot shows the ten signals after integration; the bottom plot shows the standard deviation of the integrated signal.

A MEMS vibratory gyroscope can hardly have the dynamics discussed above because the MEMS fabrication process and structure designs can easily cause mechanical structure imperfections. These imperfections account for the mismatched resonant frequency in two axes, the existence of energy dissipation terms, and uncertain values of the system parameters.

Using control techniques to compensate imperfect dynamics for the direct angle measurement is a much more challenging task than for the angular rate measurement. The reason is that it needs to achieve both the "mode matching" and "consistent vibration," while not interfering the precession of the proof mass. Very few papers have reported control algorithms to achieve that [1], [3]-[6], and they all employed some kind of energy controls to compensate the energy dissipation. A. Shkel et al. proposed a non-adaptive control strategy wherein the energy and angular momentum were feedback to control the proof mass trajectory [3]. D. Piyabongkarn et al. proposed different control algorithms for the angle and angular rate sensing [6]. However, the system stability were not theoretically proven. S. Park et al. proposed a control algorithm that consists 
of energy and mode tuning [1]. The stability is theoretically proven. But, their approach required a calibration phase prior to the normal operation of gyroscopes.
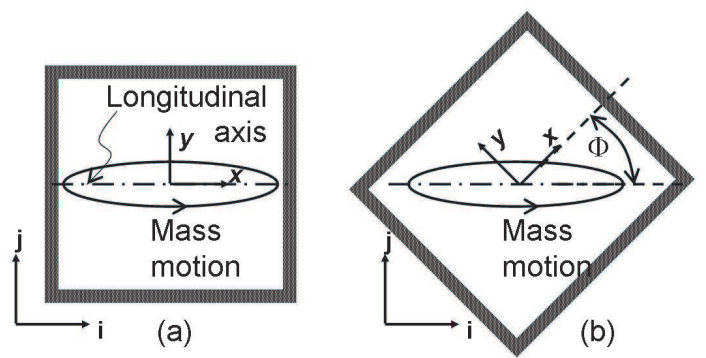

Fig. 2. Illustration of precession caused by Coriolis force. (i,j) is an inertial frame, $(\mathrm{x}, \mathrm{y})$ is the rotation frame, and $\Phi$ is the precession angle (rotation angle). (a) an initial state; (b) after 45 degree rotation.

This paper proposes a novel approach that achieves the above "ideal" dynamics using parameter estimation techniques. Furthermore, the parameter estimation is skillfully arranged so that it can be done using various existing state observer algorithms and thus benefit from their advantages. The proposed method is an one-step operation. Thus, no initialization phase is required. The design procedures of the proposed method are discussed in details. Due to the limited space in this paper, most of the mathematics proof are ignored.

\section{SyStem MODELING}

A linear vibratory gyroscope can be modeled as a springmass-damper system. Assuming that motions of proof mass are constrained in the x-y plane as shown in Fig. 2, the dynamics of a gyroscope can be modeled as follows:

$$
\begin{aligned}
m \ddot{x}+d_{x x} \dot{x}+d_{x y} \dot{y}+k_{x x} x+k_{x y} y & =u_{x}+2 m \Omega_{z} \dot{y} \\
m \ddot{y}+d_{x y} \dot{x}+d_{y y} \dot{y}+k_{x y} x+k_{y y} y & =u_{y}-2 m \Omega_{z} \dot{x}(1)
\end{aligned}
$$

where $m$ is the mass of the proof mass; $d_{x x}, d_{y y}, k_{x x}, k_{y y}$ are damping coefficients and spring constants along two principal axes; $\Omega_{z}$ is the angular rate to be measured along z-axis; $d_{x y}$ and $k_{x y}$ are the cross-axis damping coefficient and spring constant; $u_{x}$ and $u_{y}$ are the control input along $x$ and $y$ axis, respectively. The following three assumptions are made in some gyroscope control systems [7] and in this paper: (1) cross-axis terms can be neglected ( $\left.d_{x y}, k_{x y} \approx 0\right)$; (2) damping coefficients of both axes are the same $\left(d_{x x}=d_{y y}=d\right)$; (3) The mass $m$ is a known value. With above assumptions, the dynamic equation of a gyroscope can be simplified to the following:

$$
\begin{aligned}
\ddot{x}+d \dot{x}+k_{x x} x & =u_{x}+2 \Omega_{z} \dot{y} \\
\ddot{y}+d \dot{y}+k_{y y} y & =u_{y}-2 \Omega_{z} \dot{x}
\end{aligned}
$$

where $d \leftarrow d / m, k_{x x} \leftarrow k_{x x} / m, k_{y y} \leftarrow k_{y y} / m, u_{x} \leftarrow u_{x} / m$, $u_{y} \leftarrow u_{y} / m$.

Due to mechanical imperfections (both from fabrication process and structure designs), the system parameters (spring constants and damping coefficients) of a fabricated device may deviate from their designated values. Therefore, their values are assumed to be unknown in the proposed control system designs.

\section{FeEdback CONTROLS OF GYRoscope Systems}

The proposed control method is essentially a task of state feedback using estimated system parameters and estimated system dynamics. In order to do so, the dynamic equations of gyroscopes are reformulated into (3) so that unknown system parameters and system dynamics can be estimated simultaneously. Furthermore, the estimation algorithms can be chosen from various existing state observer algorithms.

$$
\begin{aligned}
\dot{X} & =f(X)+B U \\
Z & =C X+N
\end{aligned}
$$

where $N$ is the measurement noise of the gyroscope system; $X, f(X), B, U$ and $C$ are shown at top of next page. Please be noted that, in the proposed method, only the measurement of the proof mass velocity is needed to implement the feedback controller (as shown in the $C$ matrix). Also, the unknown system parameters and angular rate to be measured are all assumed to be constant. If these unknown values are timevarying, their effect can be treated as modeling error and minimized by some robust observer/controller algorithms.

The success of state estimations depends on the rank of the observability matrix. The observability matrix of a nonlinear system [8] can be obtained by the following:

$$
W_{o} \equiv \frac{\partial}{\partial X}\left[\begin{array}{llll}
Z & \dot{Z} & \ddot{Z} & \cdots
\end{array}\right]
$$

Using (4), the observability matrix of a gyroscope system (3) can be obtained as:

$$
W_{o}=\left[\begin{array}{clcc}
{\left[\begin{array}{cccc}
0 & 1 & 0 & 0 \\
0 & 0 & 0 & 1 \\
-k_{x x} & 0 & -k_{x y} & 0 \\
-k_{x y} & 0 & -k_{y y} & 0
\end{array}\right]} & {[0]_{4 \times 4}} \\
& {[0]_{4 \times 4}} & & {\left[W_{k d}\right]_{4 \times 4}}
\end{array}\right]
$$

where

$$
W_{k d}=\left[\begin{array}{cccc}
2 \dot{y} & -x & 0 & -\dot{x} \\
-2 \dot{x} & 0 & -y & -\dot{y} \\
2 \ddot{y} & -\dot{x} & 0 & -\ddot{x} \\
-2 \ddot{x} & 0 & -\dot{y} & -\ddot{y}
\end{array}\right]
$$

As long as $k_{x x} \cdot k_{y y} \neq k_{x y}^{2}$, the observability matrix in (5) is full rank. Therefore, with a proper choice of observer algorithm, one can obtain the values of those unknown parameters, angular rates, and system dynamics in real time.

\section{A. State observer design}

With system dynamic equations shown at (3), a state observer can be constructed as follows:

$$
\begin{aligned}
\dot{\hat{X}} & =f(\hat{X})+B U+L C(X-\hat{X}) \\
\hat{Z} & =C \hat{X}
\end{aligned}
$$




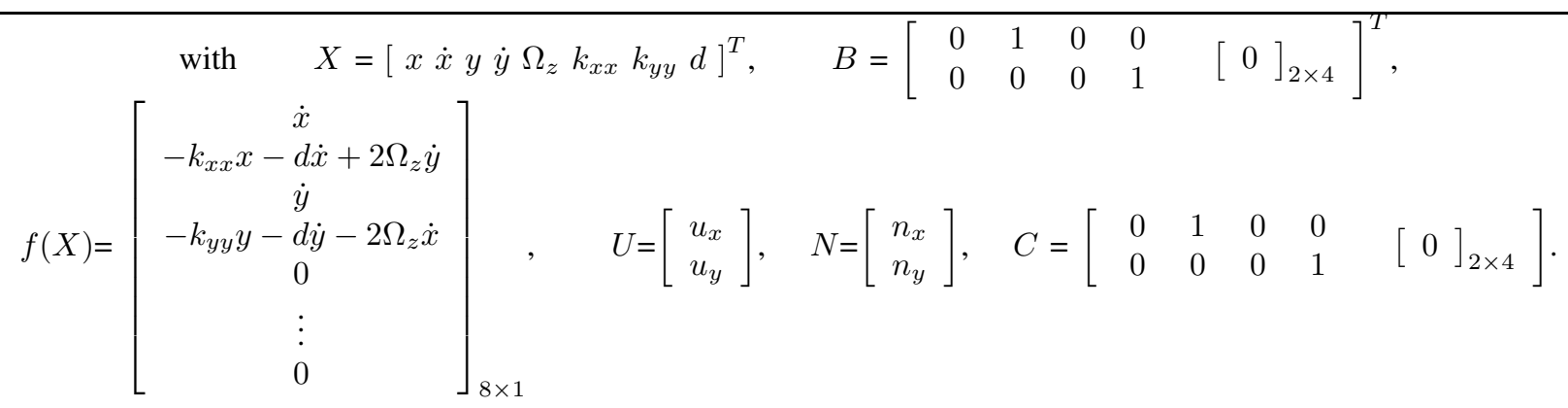

where $\hat{X}$ is the estimation of the state vector $X ; L$ is the matrix of the observer gain with a dimension of $8 \times 2$. The $L$ matrix can be divided into $L_{1}$ and $L_{2}$ matrices for the ease of equation derivations. Then, $L_{1}$ is for the estimation of system dynamics, while $L_{2}$ is for the system parameters and angular rates. To ensure the correct estimation of all states shown in (3), the $L_{1}$ and $L_{2}$ matrices can be chosen as follows:

$$
\begin{aligned}
L^{T} & =\left[\begin{array}{ll}
L_{1}^{T} & L_{2}^{T}
\end{array}\right] \\
L_{1}^{T} & =\left[\begin{array}{cccc}
0 & l_{21} & 0 & 0 \\
0 & 0 & 0 & l_{42}
\end{array}\right]_{2 \times 4} \\
L_{2}^{T} & =\left[\begin{array}{cccc}
2 \dot{\hat{y}} & -\hat{x} & 0 & -\dot{\hat{x}} \\
-2 \dot{\hat{x}} & 0 & -\hat{y} & -\dot{\hat{y}}
\end{array}\right]_{2 \times 4}
\end{aligned}
$$

where $l_{21}$ and $l_{42}$ can be any positive scalar.

As discussed above, this is not the only way to obtain the observer gain. The observer gain can be obtained by other existing observer algorithms such as extended Kalman filter (EKF) for an additional benefit of noise reduction [9].

\section{B. Feedback controller design}

To achieve the "ideal" dynamics discussed previously, the control input is designed to be the following:

$$
U=\left[\begin{array}{c}
\hat{d} \dot{\hat{x}}+\hat{k}_{x x} \hat{x}-k_{A} \hat{x} \\
\hat{d} \dot{\hat{y}}+\hat{k}_{y y} \hat{y}-k_{A} \hat{y}
\end{array}\right]
$$

where $k_{A}$ is the designated resonant frequency.

The above feedback control system can be proven to be stable by the Lyapunov 1st stability theorem. The theoretical proof is omitted here. Once the estimated state values converge to their correct values, the gyroscope dynamics can be regulated to the following dynamics:

$$
\begin{aligned}
& \ddot{x}+k_{A} x=+2 \Omega_{z} \dot{y} \\
& \ddot{y}+k_{A} y=-2 \Omega_{z} \dot{x}
\end{aligned}
$$

The above equations describe the "ideal" dynamics of a vibratory gyroscope system.

\section{Angle calculation}

According to [2], a gyroscope system that having the dynamics shown in (10), its rotation angles can be directly calculated by the following equations:

$$
\tan 2 \phi=\frac{2\left(k_{A} x y+\dot{x} \dot{y}\right)}{k_{A}\left(x^{2}-y^{2}\right)+\left(\dot{x}^{2}-\dot{y}^{2}\right)}
$$

In our approach, the gyroscope dynamics $(x, \dot{x}, y$ and $\dot{y})$ in (11) are replaced by the estimated state values $(\hat{x}, \dot{\hat{x}}, \hat{y}$, and $\dot{\hat{y}}$ ) to avoid the additional measurements of the proof mass position.

\section{Simulation RESUlts}

In a simulation case, the resonant frequencies of $\mathrm{x}$-axis and $\mathrm{y}$-axis of a fabricated gyroscope are $6.7 \%$ and $14.8 \%$ deviated from their designated values $(3.2 \mathrm{kHz})$; the measurements of the proof mass velocity along each axis are both contaminated by zero-mean white noise with the same PSDs of $2.3 \times 10^{-15}\left(\mathrm{~ms}^{-1}\right)^{2} / \mathrm{Hz}$. All the system parameters, normalized by the mass of the proof mass, are listed in Table I.

TABLE I

SYSTEM PARAMETERS AND THEIR VALUES USED IN SIMULATIONS.

\begin{tabular}{c|c}
\hline Parameters & Values (normalized) \\
\hline$\Omega_{z}$ & $4 \sin (2 \pi \times 40 t) \mathrm{rad} / \mathrm{sec}$ \\
$k_{x x}$ & $(2 \pi \times 3000)^{2} s^{-2}$ \\
$k_{y y}$ & $(2 \pi \times 3674)^{2} s^{-2}$ \\
$d$ & $200 s^{-2}$ \\
$k_{A}$ & $(2 \pi \times 3200)^{2} s^{-2}$ \\
\hline
\end{tabular}

Fig. 3 shows the trajectory of proof mass without control. The vibration of the proof diminishes because the energy dissipates through damping terms, and its trajectory is chaotic due to the frequency mismatch.

Fig. 4 to Fig. 7 show the system behaviors under control. In this case, the angular rate is a sinusoidal signal with its amplitude of $4 \mathrm{rad} / \mathrm{sec}$ and frequency of $40 \mathrm{~Hz}$. The values of observer gain $l_{21}$ and $l_{4,2}$ are both 5 . The initial guess of the state values are $10 \%$ to $20 \%$ deviated from their correct ones. In Fig. 4, it is clearly shown that the proof mass trajectory is regulated to a designated pattern wherein both the requirements of mode matching and precession are met. Fig. 5 shows the estimation of eight states, including four states from system dynamics and four states from system parameters. The estimated values converge to their correct values less than $5 \mathrm{~ms}$. However, a large chattering present in the estimated state values due to the measurement noise. Taking the estimation of angular rate as an example, the amplitude of the angular rate to be measured is $4 \mathrm{rad} / \mathrm{sec}$ and its estimated values oscillates between -150 to $150 \mathrm{rad} / \mathrm{sec}$ ( Fig. 6). For comparison, the rotation angle is calculated in 

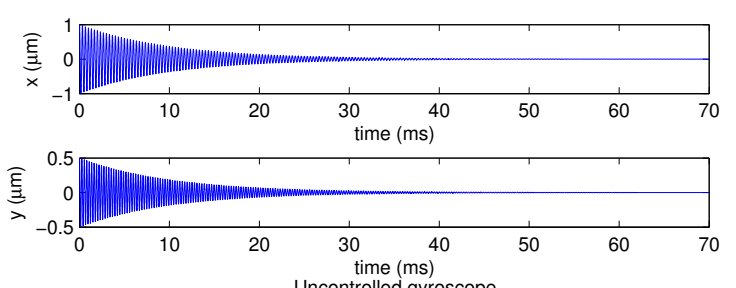

Uncontrolled gyroscope

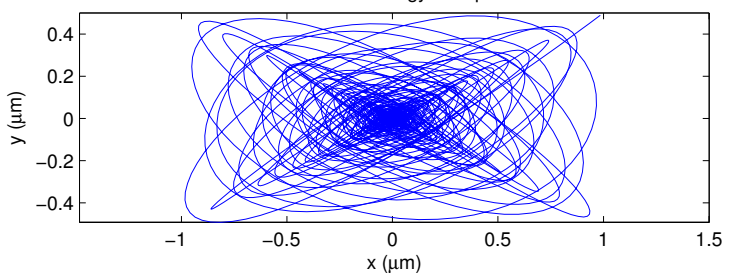

Fig. 3. Trajectory of the proof mass without controls.

two ways: integrating the estimated angular rates; using the equations shown in (11). As shown in Fig. 7, the proposed method shows much better accuracy (within 0.5 degree) than the integration one.

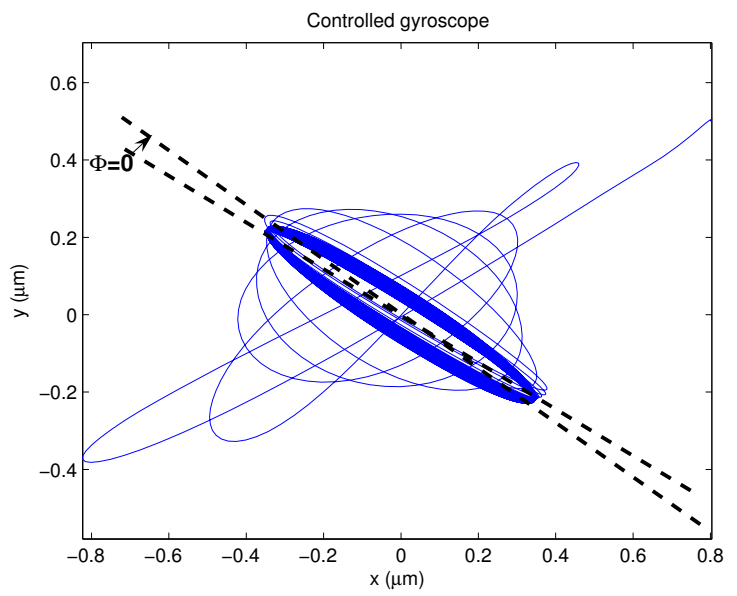

Fig. 4. Trajectory of the proof mass under controls. Angular rates are timevarying as $4 \sin (2 \pi \times 40 t) \mathrm{rad} / \mathrm{sec}$.

\section{Discussion}

According to the simulation results (Fig. 7), obtaining the rotation angles by the equation (11) does show a better accuracy than by the integration method when the measured signals are contaminated by noise. However, the equation is applicable only when the employed gyroscope have the dynamics described in (10). This dynamics (signal variations) may be inadequate for parameter identifications and thus set the limitation on the approaches that employ parameter identifications.

In this paper, the cross-axis damping coefficients and spring constant are both neglected, and the damping coefficient along two axes are assumed to be the same. These assumptions may
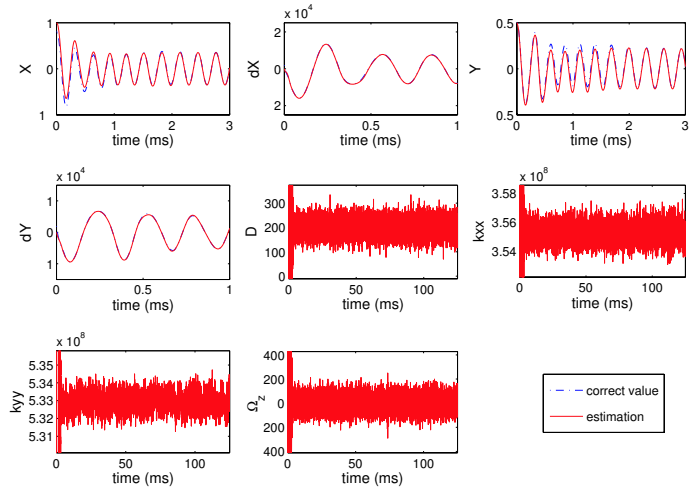

Fig. 5. Estimation of system state values. These values are shown in nondimensional units.

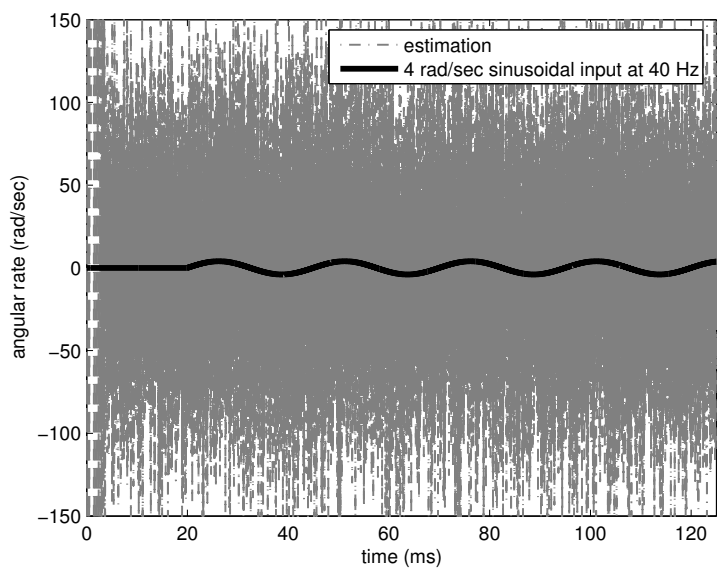

Fig. 6. Estimation of the angular rates.

be impractical in most cases. Our research indicates that, in theory, all the system parameters in (3) (7 unknowns) can be correctly estimated by the proposed method. However, the observability matrix is close to singularity due to the value of the angular rate is relative small compared to other system parameters. The numerical error fails the entire state estimations.

Fig. 4 shows the amplitude of the proof mass vibration is less than $1 \mu \mathrm{m}$, a bit less than its designated maximum value. This can be attributed to the energy dissipation during the transient response of the parameter identification. A smaller vibration amplitude would lead to a smaller vibration speed, in turns, it decrease the sensing accuracy of gyroscopes. Therefore, the transient response time should be kept as small as possible in the proposed method. However, it is rather difficult to do so by selecting the proper observer gain. On the other hand, the diminished vibration amplitude can be compensated by the controller designed. Our research shows that this can be done if the measurements of the gyroscope system include both the position and velocity of the proof mass. 

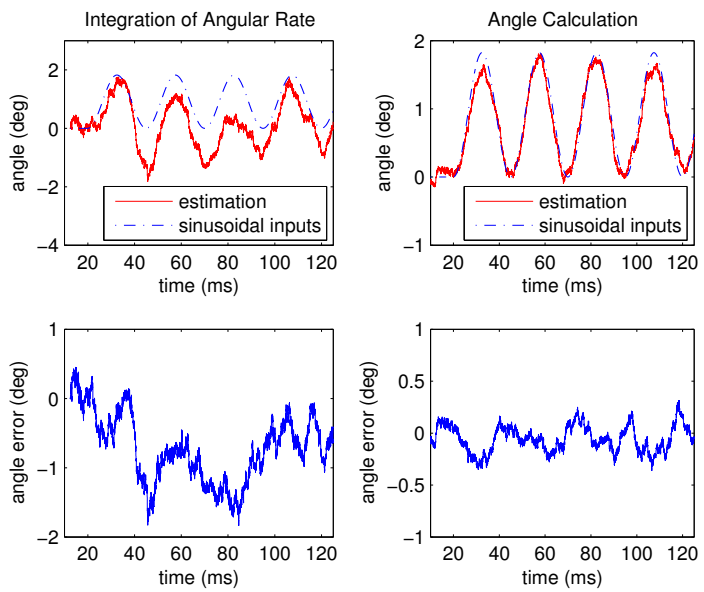

Fig. 7. The rotation angles are calculated in two ways: integrating the angular rate over time; the direct angle measurements.

\section{CONCLUSION}

This paper presents a control algorithm that uses velocity feedback only. With this control method, the proof mass of a gyroscope can oscillate along two axes at the same resonant frequency while preserving its precession motions, even when the mechanical structure uncertainties are present. Under this regulated dynamics, the rotation angles are calculated using estimated state values. Simulation results indicate that the rotation angle obtained by this method is more accurate than by the method of integrating the angular rates. However, the oscillation amplitude can decrease a bit which could decrease the sensing accuracy of angular rates.

In a demonstrating case, the mechanical structure uncertainties caused $10 \% \sim 20 \%$ parameter variations; the measured velocity signals are contaminated by zero-mean white noise with a PSD of $2.3 \times 10^{-15}\left(\mathrm{~ms}^{-1}\right)^{2} / \mathrm{Hz}$; the angular rate to be measured is $4 \sin (2 \pi \times 40 t) \mathrm{rad} / \mathrm{sec}$. The proposed algorithm can obtain the rotation angle with an accuracy less than 0.5 degree.

\section{REFERENCES}

[1] S. Park, R. Horowitz, and C.-W. Tan, "Dynamics and control of a MEMS angle measuring gyroscope," Sensors and Actuators A, Vol. 144, Issue 1, pp. 56-63, 2008.

[2] B. Friedland and M. Hutton, "Theory and Error Analysis of Vibratingmember Gyroscope," IEEE Transactions on Automatic Control, Vol. AC23, No. 4, pp. 545-556, 1978.

[3] A. Shkel, R. Horowitz, A. Seshia, S. Park, and R. Howe, "Dynamics and Control of Micromachined Gyroscopes," Proceedings of the American Control Conference, pp. 2119-2124, 1999.

[4] C. Painter and A. Shkel, "Detection of Orientation and Predicted Performance of a MEMS Absolute Angle Measuring Gyroscope," Proceedings of the 4th International Workshop on Structural Health Monitoring, pp. 1011-1018, 2003.

[5] C. Painter and A. Shkel, "Experimental evaluation of a control system for an absolute angle measuring micromachined gyroscope," IEEE Conference on Sensors, pp. 1084-1087, 2005.

[6] D. Piyabongkarn, R. Rajamani, and M. Greminger, "The development of a MEMS gyroscope for absolute angle measurement," IEEE Transactions on Control Systems Technology, Vol. 13, No. 2, pp. 185-195, 2005.
[7] R. P. Leland, "Adaptive mode tuning for vibrational gyroscopes," IEEE Transactions on Control Systems Technology, vol. 11, no. 2, pp. 242-247, 2003.

[8] M. Vidyasagar, Nonlinear Systems Analysis, Prentice Hall, 1993.

[9] C.-Y. Chi, Y.-P. Peng, and T.-L. Chen, "Compensation of interface circuit errors for MEMS gyroscopes using state observers," The 3rd IEEE International Conference on Sensing Technology (ICST 2008) (ISBN: 978-1-4244-2176-3), Tainan, Taiwan, Nov. 30th - Dec. 3rd, pp. 25-30, 2008 . 